\title{
Metallicity effects in Mira variables: a model study
}

\author{
M. Scholz \\ Institut für Theoretische Astrophysik der Universität Heidelberg, \\ Tiergartenstr. 15, 69121 Heidelberg, Germany, and School of Physics, \\ University of Sydney, Sydney NSW 2006, Australia \\ P.R. Wood \\ Australian National University, Research School of Astronomy and \\ Astrophysics, Weston Creek ACT 2611, Australia
}

\begin{abstract}
M-type Mira models with a moderate deviation from solar metallicity $(2 \times$ and $0.5 \times)$ have been computed and compared to solar-metallicity models. Selected effects of abundance changes on pulsation properties, atmospheric stratifications, spectral features (colours) and surface brightness distributions are discussed.
\end{abstract}

\section{Introduction}

Dynamic model atmospheres of oxygen-rich Mira variables have so far only been computed for a solar-type element mixture (e.g. Bessell, Scholz \& Wood 1996 [BSW96]; Hofmann, Scholz \& Wood 1998 [HSW98]; Woitke et al. 1999; Höfner et al. 2003; Tej et al. 2003 [TLSW03]) although the strong influence of molecular band absorption of, for example, TiO and $\mathrm{H}_{2} \mathrm{O}$ upon the temperature stratification is expected to result in a substantial metallicity dependence of the atmospheric structure. Bessell et al. $(1989,1991$, both papers henceforth called BBSW89/91) found and discussed significant metallicity effects in models of non-pulsating $\mathrm{M}$ giants.

We constructed for this exploratory study a small set of M-type Mira model atmospheres with $2 \times$ and $0.5 \times$ solar metallicity. In a first step, we kept the density structure of the pulsation models of HSW98 and TLSW03 and only re-calculated the temperature stratification for the new molecule mixture and new absorption coefficients of the non-solar element composition. In a second step, consistent metal-poor pulsation and atmospheric models were computed for the same basic parameters (mass $M$, luminosity $L$, pulsation period $P$ ) as the P model series of HSW98/TLSW03. We then compared selected properties of these new models with those of the models of HSW98/TLSW03.

\section{Models}

Our exploratory model series is based on the P and M series of HSW98/TLSW03. We use the abbreviated designations of TLSW03: P10, for example, means the 
$\mathrm{P}$ series model with visual phase $1+0.0$. P10p and P10m means the P10 pulsation model whose atmospheric metallicity $Z$ is enhanced $(2 \times)$ and reduced $(0.5 \times)$, respectively, compared to the solar value $\mathrm{Z}_{\odot}$ in $\mathrm{P} 10$, and whose atmospheric temperature stratification was re-calculated for these modified element compositions. The non-pulsating parent star of the $\mathrm{P}$ series has $M=1.0 \mathrm{M}_{\odot}$, $L=3470 \mathrm{~L}_{\odot}, P=332 \mathrm{~d}$ and Rosseland radius $R_{\mathrm{p}}=241 \mathrm{R}_{\odot}$ (defined as the distance $r$ from the star's centre to where the Rosseland optical depth $\tau_{\text {Ross }}$ equals unity). For the $\mathrm{M}$ series parent star, the values are $1.2 \mathrm{M}_{\odot}, 3470 \mathrm{~L}_{\odot}, 332 \mathrm{~d}$ and $260 \mathrm{R}_{\odot}$. The parent star of the new metal-poor $\left(0.5 \times \mathrm{Z}_{\odot}\right) \mathrm{H}$ model series was chosen to have the same $M, L$ and $P$ (335d) as that of the $\mathrm{P}$ series, and its Rosseland radius becomes $226 R_{\odot}$. Note that the non-pulsating parent star does not really exist but its Rosseland diameter may still be used as a fundamental parameter of the real Mira star (cf. the discussion in Scholz 2003). The "surface" layer of the model atmosphere (cf. HSW98) is at $5 R_{p}$ for the M and P series and at $4 R_{p}$ for the new $\mathrm{H}$ series. A detailed description of the $\mathrm{H}$ models will be given in a forthcoming paper (Lançon, Scholz \& Wood, in preparation).

We constructed pre-maximum model atmospheres P08p, P08m, M08p and M08m and near-maximum atmospheres P10p, P10m, M10p and M10m. Models around phases $\mathrm{n}+0.8$, when the new shock front emerges from deep layers, are found to have relatively small Rosseland radii $R$, to have high effective temperatures $T_{\text {eff }} \propto\left(L / R^{2}\right)^{1 / 4}$ despite lower $L$ than at maximum and to exhibit particular spectral properties (TLSW03). The P model series shows remarkable cycle-tocycle variations. For instance, the near-maximum model P20 shows excessive strengths of deep molecular bands and severe molecular-band contamination of near-continuum bandpasses (HSW98; Jacob \& Scholz 2002), whereas samephase models of three other cycles investigated by HSW98/TLSW03 including our $\mathrm{Z}_{\odot}$ reference model $\mathrm{P} 10$ are much less conspicuous.

The new metal-poor $\mathrm{H}$ series of pulsation models shows noticeably smaller cycle-to-cycle variations than the equivalent $\mathrm{P}$ series. Bolometric light curves and dynamic structures repeat more regularly than in the $P$ series in four successive cycles which we checked in some detail. This higher regularity is also found when the mass increases from 1.0 to $1.2 \mathrm{M}_{\odot}$ (P vs. M models; HSW98). We constructed atmospheric models for only one cycle (H082, H09, H10) in this exploratory study.

Effective temperatures of models $\mathrm{P} 08 \mathrm{p} / \mathrm{P} 10 \mathrm{p}$ and $\mathrm{P} 08 \mathrm{~m} / \mathrm{P} 10 \mathrm{~m}$ are slightly lower and higher, respectively, than those of their counterparts P08 and P10 but differences do not exceed $40 \mathrm{~K}$. Systematic $T(r)$ and $T\left(\tau_{\text {Ross }}\right)$ stratification differences are modest and hardly outside the range of cycle-to-cycle differences in the solar-metallicity $\mathrm{P}$ models, the range of differences caused by relatively small changes in stellar mass (say, 1.0 to $1.2 \mathrm{M}_{\odot}$ ) and the range of the effects of uncertainty of phase assignment (cf. TLSW03). Similarly, $T_{\text {eff }}$ differs little between H082/H10 and P08/P10. Deviations of density and temperature stratifications are significant but again not clearly beyond the range of cycle-to-cycle variations of the $\mathrm{P}$ series. 


\section{Spectra and disk brightness distribution}

First-glance inspection of the 0.5 to $4 \mu \mathrm{m}$ spectrum shows a noticeable to sometimes striking strengthening/weakening of medium-strong to strong molecular bands of $\mathrm{TiO}$ and $\mathrm{H}_{2} \mathrm{O}$ with increasing/decreasing metallicity. But, as expected from the above-outlined effects of $\mathrm{Z}$ on the atmospheric temperature stratification, cycle-to-cycle differences of the solar-metallicity $\mathrm{P}$ models may be of a similar order or even more conspicuous.

According to BBSW89/91, colour indices of near-continuum bandpasses may be sensitive to variations of $Z$ in non-pulsating $M$ giants. The $(J-H)$ vs. (H-K) diagram shows a clear separation of different metallicities for effective temperatures above about $3000 \mathrm{~K}$. Models are shifted towards smaller $(\mathrm{J}-\mathrm{H})$ and larger $(\mathrm{H}-\mathrm{K})$ when $\mathrm{Z}$ increases. Effects are of the order of 0.05 to $0.15 \mathrm{mag}$ for a metallicity change by a factor of 3 and, thus, accessible to high-accuracy photometry only. The effects of changing surface gravity and atmospheric extension (X vs. Z model series of BBSW89/91) are smaller though still significant. Solar-metallicity Mira models of BSW96 and HSW98/TLSW03 populate a fairly narrow strip in this diagram even without sorting for phases, and agree satisfactorily with observed stars (Lançon et al., in preparation).

When the $Z_{\odot}$ models of the $P$ and $M$ series with phases $n+0.8$ and $n+0.0$ are entered in the $(\mathrm{J}-\mathrm{H})$ vs. $(\mathrm{H}-\mathrm{K})$ diagram, their positions occupy the approximate range of the static $X$ and $Z$ series of BBSW 89/91, except M10 and M20 that are much cooler $\left(T_{\text {eff }}=2750 \mathrm{~K}\right.$ and $\left.2650 \mathrm{~K}\right)$ than $3000 \mathrm{~K}$ and $\mathrm{P} 20$ that shows extreme contamination of near-continuum bandpasses (e.g. Jacob \& Scholz 2002). Increasing and decreasing $\mathrm{Z}$ in the stellar atmosphere by a factor of 2 shifts the position of the $\mathrm{Z}_{\odot}$-entries $\mathrm{P} 08, \mathrm{M} 08$ and $\mathrm{P} 10$ in a direction and by a magnitude that is in reasonable agreement with predictions estimated from the BBSW89/91 non-Mira models. Cycle-to-cycle variations (excluding the extreme $\mathrm{P}$ series cycle with P18 and P20) are substantial but do not mask metallicity differences. As one compares the positions of the solar-composition $1.2 \mathrm{M}_{\odot} \mathrm{M}$ models with those of the $1.0 \mathrm{M}_{\odot} . \mathrm{P}$ models at phases $n+0.8$ (excluding $\mathrm{P} 18$ ), they appear essentially shifted upward by roughly $0.1 \mathrm{mag}$ in $(\mathrm{J}-\mathrm{H})$ only. Hence, though the shifting direction is different from that of decreasing Z, mass effects severely interfere with metallicity effects in this diagram. Perhaps still more worrying, the position of the fully consistent (i.e. both pulsation and atmospheric model computed with identical Z) metal-poor H082 model is very close to M08 in this 2-colour diagram rather than close to $\mathrm{P} 08 \mathrm{~m}$. Near maximum, on the other hand, the position of $\mathrm{H} 10$ is very close to $\mathrm{P} 10 \mathrm{~m}$.

This and more extensive studies (Lançon et al., in preparation) of spectral properties indicate that there is no obvious easy way to determine the metallicity of an M-type Mira field star, at least for moderate deviations (factor of the order of 2) from $\mathrm{Z}_{\odot}$. Rather, suitable combinations of spectral features have to be found that can disentangle effects of varying metallicity from those of varying mass and those of phase and cycle dependence of the star's structure.

Finally, we comment on the model-predicted brightness distributions on the stellar disk at selected wavelengths. It turned out that the influence of metallicity may be quite substantial. In particular, the effects of $Z$ upon molecular-band contamination in near-continuum bandpasses in the near infrared may affect the interferometric measurement of continuum diameters and therefore the estimates 
of Rosseland diameters of the Mira variable and its parent star (cf. Jacob \& Scholz 2002; Scholz 2003).

Acknowledgments. This research was in part supported by the Australian Research Council and the Deutsche Forschungsgemeinschaft within the linkage project "Red Giants".

\section{References}

Bessell, M.S., Brett, J.M., Scholz, M., Wood, P.R. 1989, A\&AS, 77, 1 (BBSW89/91); erratum: A\&AS, 87, 621

Bessell, M.S., Brett, J.M., Scholz, M., Wood, P.R. 1991, A\&AS, 89, 335 (BBSW89/91)

Bessell, M.S., Scholz, M., Wood, P.R. 1996, A\&A, 307, 481 (BSW96)

Hofmann, K.-H., Scholz, M., Wood, P.R., 1998, A\&A, 339, 846 (HSW98)

Höfner, S., Gautschy-Loidl, R., Aringer, B., Jørgensen, U.G. 2003, A\&A, 399, 589

Jacob, A.P., Scholz, M. 2002, MNRAS, 336, 1377

Scholz, M. 2003, in SPIE Conf. Ser, 4838, Proceedings in Astronomical Telescopes and Instrumentation 2002, Interferometry for Optical Astronomy II, ed. Traub, W.A. (Bellingham: SPIE), 163

Tej, A., Lançon, A., Scholz, M., Wood, P.R. 2003, A\&A, in press (TLSW03)

Woitke, P., Helling, Ch., Winters, J.M., Jeong, K.S. 1999, A\&A, 348, L17

\section{Discussion}

Kiss: Is there any suggestion that metallicity may affect the light curve shape?

Scholz: Yes, the light curve of the low-metallicity $\mathrm{H}$ model series looks noticeably different from that of the $\mathrm{P}$ series and shows smaller cycle-to-cycle variations. 